\title{
Performance Analysis and Throughput Enhancement of the STET Technique for WLAN IEEE 802.11ad
}

\author{
M. Vanitha ${ }^{1, *}$, J. Kirubakaran ${ }^{2}$ and K. Radhika ${ }^{2}$ \\ ${ }^{1}$ Department of Electronics and Communication Engineering, Saveetha Engineering College, Chennai, 602105, India \\ ${ }^{2}$ Department of Electronics and Communication Engineering, Muthayammal Engineering College, Rasipuram, 637408, India \\ ${ }^{*}$ Corresponding Author: M. Vanitha Email: vanitha@saveetha.ac.in \\ Received: 06 February 2021; Accepted: 18 April 2021
}

\begin{abstract}
The IEEE 802.11ad innovation has enabled the impact of remote devices in unauthorized $60 \mathrm{GHz}$ unlicensed frequency band at Giga bits per second information transfer rate in speed concentrated $5 \mathrm{G}$ applications. We have presented an innovative work that deals with the upgradation of the ability of IEEE 802.11ad wireless LAN to make it suitable for wireless applications. An exact examination on the IEEE 802.11ad analysis has been carried out in this work to achieve the greatest throughput. This has pulled attraction in broad consideration for accomplishing the pinnacle transmission rate of $8 \mathrm{Gbit} / \mathrm{s}$. IEEE $802.11 \mathrm{ad}$ is a convention utilized for extremely high information rates (around $8 \mathrm{Gbit} / \mathrm{s}$ ) and for short range remote correspondence of around 1 to 10 meters. The Multi-layer with Multi-User Multiple Input Multiple-Output (MLMU-MIMO) innovation has enabled transmission of multiple information packets all the while from the WLAN IEEE 802.11 ad Access Point (AP) to various receivers by means of different wireless applications. To help the MLMU-MIMO innovation at Medium Access Control (MAC), a new method called Simultaneous Transmission for Enhanced Throughput (STET) has been presented for enhancement of the transmission rate of the wireless standard. A mathematical model has been used for the evaluation of the performance of an IEEE 802.11ad AP empowering the STET allocation technique. This STET model can help determination of the throughput improvement of a STET technique, for example, audio information, images, good quality and foundation impact. The main goal of this work is to analyze the improvement of the STET allocation technique in regard to the usage of rare frequency band, when obtaining spectrum access between the various access points. The theoretical and simulation outcomes are also confirmed by Riverbed modeler 17.5 simulation tool.
\end{abstract}

Keywords: WLAN; STET; MAC

\section{Introduction}

With up to $7 \mathrm{GHz}$ unlicensed data transmission in many nations, 60-GHz millimeter-wave (mm-wave) has shown incredible potential in remote individual zone systems (WPANs) and remote neighbor of territory

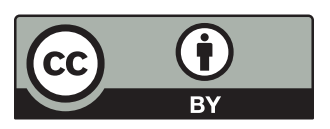

This work is licensed under a Creative Commons Attribution 4.0 International License, which permits unrestricted use, distribution, and reproduction in any medium, provided the original work is properly cited. 
systems (WLANs) [1]. As needs be, here progressing endeavors in understanding the upcoming age of portable remote systems like $5 \mathrm{G}$ has been a requirement for high pinnacle execution markers (i.e., multiple times superior to the current 4G) [2,3]. IEEE 802.11ad WLAN licenses are required for accomplishment of an improved throughput up to $8 \mathrm{Gbps}$ on recurrence channels. Specifically, the 802.11ad amendment for the information rates differ from $385 \mathrm{Mbps}$ to $8085 \mathrm{Mbps}$ [4]. The improved throughput is subject to this recent upgradation, considering the possibility of the critical increment of exchange information by the 802.11ad standard [5]. Multi-User Multiple-Input Multiple-Output (MUMIMO) innovation is the major element that permits the arrival of 802.11ad at the $8 \mathrm{Gbps}$ information rate. No doubt, MU-MIMO is characterized as a plan where distinctive base stations each with possibly different receiving wires, transmit and get all the while free information streams. The type of MU-MIMO in the IEEE 802.11ad standard is the Down-Link (DL) MUMIMO. Hence, the 802.11ad passage stations having various radio wires can transmit a few data to various endorser stations simultaneously over an identical recurrence band [6]. Multiclient MIMO transmission at the MAC layer level has been proposed as a directing guideline for empowerment of down connection. At that point, the designation conspire is utilized for the IEEE 802.11ad determinations [7,8].

\section{Related Work}

The STET strategy stretches out the current STET system to down-interface various information casings to different destinations. Actually, the STET method was presented in the WLAN IEEE 802.11 standard by the 802.11e WiFi in 2006 [9-11]. The objective of this method is to provide enhanced service quality to the remote LAN frameworks. In setting another such system, to be specific, Enhanced Throughput Multiclient Channel Access (ETMCA) STET, was proposed. It enables channel access to a particular endorser to transfer a combat free period (CFP) during a limited interval. Consequently, a particular endorser can transmit as much of information as could be expected under the circumstances, as the farthest point of its STET isn't surpassed. In any case, a portion of the current STET rules become obsolete and not in use with the reception of DL MU-MIMO in 802.11ad [12,13]. In reality, these standards expect one-on-one transmission enabling just a single explicit supporter to win the interior challenge and transmit during the won STET, which does not adjust to the down connection multiclient MIMO point [14-16]. 802.11 has embraced the STET procedure for helping the down-connection multi-client MIMO communication [17,18]. Typically, an ETMCA STET has two levels in the recent 802.11 standard: the commencement of the STET (which happens at the point when the ETMCA rules grant access to the channel) and the various information transmission inside a STET (which happens at the point whenever an ETMCA Function (ETMCAF) holds the privilege to get to the channel). Though in the 802.11ad standard, the commencement level is equivalent to the present wifi, the various information transmission levels have been upgraded to permit synchronous information of different forms of trade successions. In this specific situation, another level has been combined with the ETMCA STET [19,20]. It happens after the commencement level, furthermore, before the various information transmission level. In the offer level, data might be transferred from an auxiliary channel if the essential access channel shares its STET, irrespective of whether the back-off counter for that optional access channel have not been at zero [21-23]. The fundamental working standards of the STET Sharing strategy, for example, each ETMCAF of a passageway goes after STET has utilized all its known ETMCA attributes, as it does in the present 802.11ad. When an ETMCA owns a STET, it turns into the possessor of that STET. Furthermore, its comparable supporter channel turns into the essential access channel, while different other access channels become optional access channels. The essential access channel can, at that point, choose whether or not to impart its STET to the optional access channel for synchronous transmissions [24,25]. On the off chance that it does, the won STET turns into a Different Subscriber STET (DS-STET). 
The essential access channel likewise can choose the auxiliary access channel as desired, to impart with the won STET and which has the goal of focusing for transmissions.

Further, the length of the STET is inspected by the STET point of confinement of the essential access channel while the information period is examined by the measure of information planned to be transferred by the essential channel. When the essential channel has completed its transmission, the DS-STET is finished, regardless of whether or not the optional channel has data to transmit [26]. In this work, we propose a numerical model for the STET sharing method empowered at the 802.11ad passageway. We are of the view that STET portion procedure speaks to the special MAC edge of the IEEE 802.11ad change, permitting the accomplishment of multiclient MIMO communication at the layer. In view of the presented yield, we determine an expository analysis for the computation of the upgraded throughput of a given access channel. While the presented pattern is an expansion of the outstanding existing pattern [27-29] and the entirety of the current numerical patterns created to investigate the ETMCA STET, its primary curiosity comprises the joining of the STET channel assignment model presented by the $802.11 \mathrm{ad}$. When required, the effect of the STET portion procedure on the feasible throughput of every channel can be broken down and subsequently feature the upgraded throughput accomplished regarding range usage. Recurrence channel results in further coordination for the sensibility between the distinctive channels. The exactness of the presented scientific model and the accomplished simulation outcomes are verified by Riverbed modeler 17.5 simulator tool. This paper has been organized as follows: A description of the related work of mathematical model for an IEEE 802.11ad access point to activate the ETMCA STET allocation mechanism has been provided in Section 2. Section 3 deals with the analysis of the impact of IEEE 802.11 ad with the STET allocation system on the enhanced throughput. Section 4 discusses the performance analysis of IEEE 802.11ad with STET. Section 5 concludes this paper.

\section{Modeling of IEEE 802.11ad with STET Allocation Mechanism}

In this section, we have presented a mathematical model for IEEE 802.11ad access point empowering the STET allocation mechanism to enable calculation of the transmission probability $\mathrm{P}_{\mathrm{r}}(\mathrm{n})$ for every one of its $\mathrm{A}(\mathrm{n})$ (where $\mathrm{n} \in[V, I, E, K]$ ). At that point, utilizing the computed $\mathrm{P}_{\mathrm{r}}(\mathrm{n})$, we examine the various occasions that can be witnessed inside the channel communication focusing at developing an analytical model for the inference of the enhanced throughput $N[\mathrm{n}]$ of each $A[\mathrm{n}]$. Fig. 1 shows the structure of IEEE 802.11ad model.

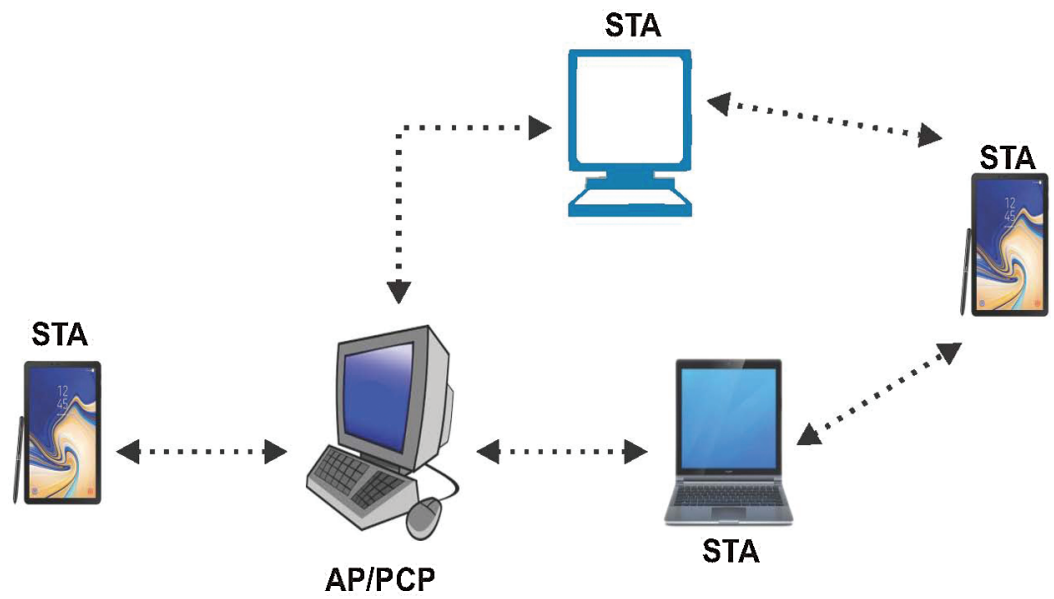

Figure 1: Structure of IEEE 802.11ad 


\subsection{Attributes of 802.11ad Mathematical Model}

Before specifying the presented mathematical model, we start by exhibiting a list of attributes about speculation. The list of attributes given in Tab. 1 considers $\mathrm{P}(\mathrm{n})$ as the probability of discovery of the channel occupied for an $\mathrm{A}[\mathrm{n}]$ and $\mathrm{P}_{\mathrm{r}}(\mathrm{sh})$ as the probability to share a STET between the entrance channels. The transmission line of each $\mathrm{A}[\mathrm{n}]$ has been assumed to be under consistent immersion conditions enabling the arrival at the upgraded throughput of an $\mathrm{A}[\mathrm{n}]$. The crash likelihood $\mathrm{P}_{\mathrm{co}}[\mathrm{n}]$ of an $\mathrm{A}[\mathrm{n}]$ is consistent and autonomous from the extent of retransmissions. It is the key estimate in the existing scientific model which confirms the STET in the proposed model.

Table 1: Attributes of 802.11ad analytical model

\begin{tabular}{ll}
\hline Attributes & Description \\
\hline $\max [\mathrm{n}]$ & Maximum back-off stage of the $A[\mathrm{n}]$. \\
$W_{\min }[\mathrm{n}]$ & Minimum contention window of the $A[\mathrm{n}]$. \\
$W_{\max }[\mathrm{n}]$ & Maximum contention window of the $A[\mathrm{n}]$. \\
$C W_{i}[\mathrm{n}]$ & Contention window size at back-off stage $i$. \\
$\mathrm{N}[h]$ & Number of ADUs of the $A C[h]$ in its STET[h]. \\
$A D U$ & ADU length \\
$T_{A D U}$ & Transmission time of an ADU. \\
$T_{p h}$ & Time duration of the PHY header \\
$T_{b a}$ & Transmission time of a Block Acknowledgment. \\
$T_{a i f s}$ & Time of an arbitration inter-frame space \\
$T_{s i f s}$ & Time of a short inter-frame space \\
$\delta$ & Time of a signal propagation \\
$\sigma$ & An empty slot time. \\
\hline
\end{tabular}

\subsection{Transmission Probability $\boldsymbol{P}_{\boldsymbol{r}}(\boldsymbol{n})$}

Let $S_{j}(n)$ be the stochastic procedure speaking to the back-off range $(j=1,2 \ldots, N[n])$ of the $A[n]$ at period $n$. Let $b_{k}(n)$ be the stochastic procedure speaking to either the back-off time counter $(k=0,1,2 \ldots$, $\left.\mathrm{W}_{\mathrm{k}}[\mathrm{n}]\right)$ or the $\mathrm{k}^{\text {th }}$ transmitted ADU $(\mathrm{m}=0,-1, \ldots, \mathrm{T}[\mathrm{n}]+1)$ of the $\mathrm{A}[\mathrm{n}]$ at period $\mathrm{t}$ where, the $\mathrm{W}_{\mathrm{k}}[\mathrm{n}]$ and the $\mathrm{T}[\mathrm{n}]$ are given as

$W_{k}[\mathrm{n}]=\operatorname{Minimum}\left(W_{k}[\mathrm{n}], 2 j * W_{0}[\mathrm{n}]\right)$

$T[\mathrm{n}]=\operatorname{STET}(\mathrm{n}) / T_{p h}+T_{A D U}+2 *\left[T_{s i f s}\right]+T b a+2 \delta$

\section{Performance Analysis of IEEE 802.11 ad with STET}

In an IEEE 802.11ad association, physical layer of each end executes a pole confining instrument for shaping directional bars using a staged exhibit receiving wire. PHY layers in the standard are of four types, all of which have reinforced enormous MCSs. In the earlier adjustment, the channel list for IEEE 802.11ad movement in the frequency band has recollected four channels as shown in Tab. 2. 
Table 2: IEEE 802.11ad allocation bandwidth

\begin{tabular}{llll}
\hline 802.11 ad allocation Channel & Center frequency & Maximum frequency & Bandwidth in $\mathrm{GHz}$ \\
\hline 2 & $116.64 \mathrm{GHz}$ & $118.8 \mathrm{GHz}$ & $4.32 \mathrm{GHz}$ \\
4 & $120.96 \mathrm{GHz}$ & $123.12 \mathrm{GHz}$ & $4.32 \mathrm{GHz}$ \\
6 & $125.28 \mathrm{GHz}$ & $127.44 \mathrm{GHz}$ & $4.32 \mathrm{GHz}$ \\
8 & $129.6 \mathrm{GHz}$ & $131.76 \mathrm{GHz}$ & $4.32 \mathrm{GHz}$ \\
\hline
\end{tabular}

The $60 \mathrm{GHz}$ connect has an issue with blockage helplessness. Besides, there is the likelihood of the reception apparatus course changing its activity. Thus, the connection upkeep is imperative for the effective development of IEEE 802.11ad on Wi-Fi systems.

\subsection{IEEE 802.11ad with STET}

In this IEEE 802.11ad with STET, the maximum throughput for the various access points of an IEEE 802.11ad, which empowers the STET allocation technique has been examined. The mathematical outcomes achieved have been confirmed by simulation. The ETMCA work with STET allocation has been executed in a uniquely crafted Riverbed modeler 17.5 simulator. The primary inspirations are: the plausibility of detaching the 802.11ad layer analysis from the remainder of system and the quicker analysis of simulations. Fig. 2 shows the throughput heterogeneity of a given access point as indicated by the possibility of allocation. We comment that, the throughput of every access point increments with the expansion of the possibility of allocation. STET allocation technique enables the transfer of various access points for an access point offered.

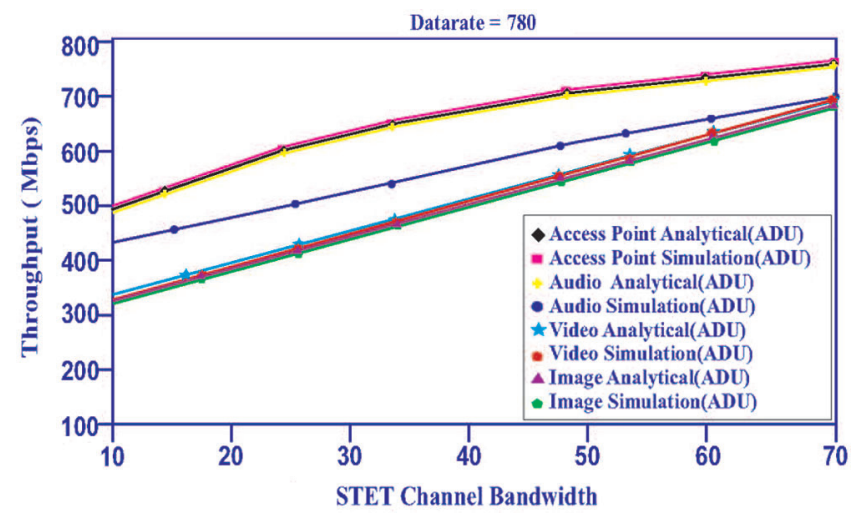

Figure 2: Throughput vs STET channel bandwidth for channel data rate $=780$

Finally, analysis made using Fig. 3 shows that, when the possibility of STET allocation is high, the throughput of all access points associates to the same value, in the light of the fact that, the access point transmits whenever guaranteed access point is associated with the frequency spectrum. Extremely high throughput proves that the quantity of utilized reception as high. Hence, there is a probability of proficient exploitation from maximum spatial reuse for maximized throughput.

\subsection{Maximum Throughput using STET Allocation}

Fig. 4 shows the maximum magnitude of STET throughput with different numbers of subscribers. Figure shows the maximum throughput using STET allocation technique getting a better performance 
than by the existing allocation technique when the subscriber number is minimum and attains the same performance as STET channel allocation for a large number of subscribers. Also, STET is initially superior and then inferior to channel allocation with increase in the number of subscribers.

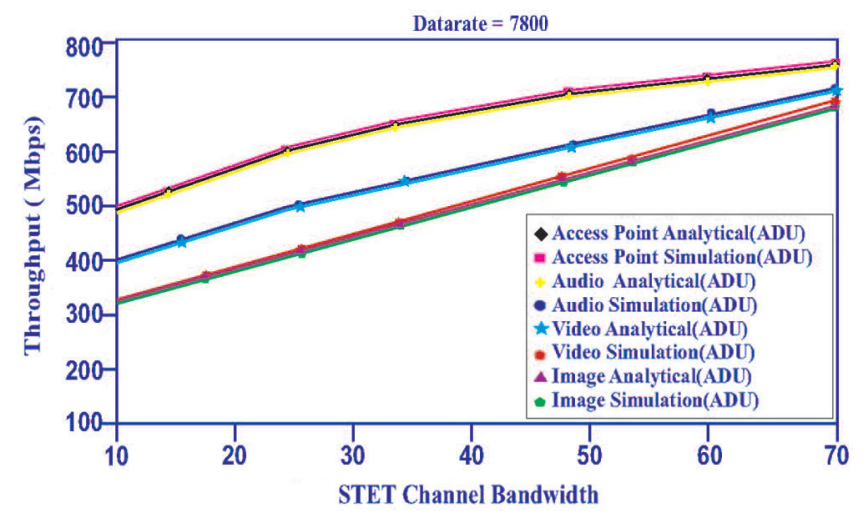

Figure 3: Throughput vs STET channel bandwidth for channel data rate $=7800$

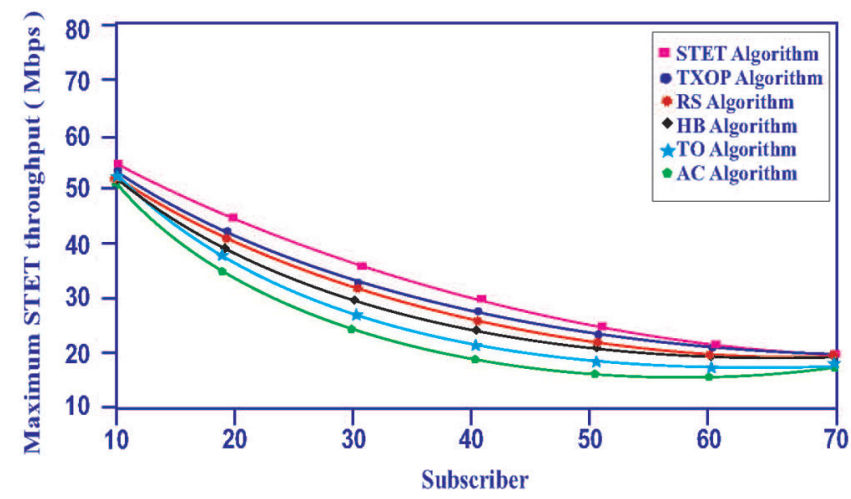

Figure 4: Maximum STET throughput vs. subscriber

Fig. 5 shows the minimum of STET throughput with different numbers of subscribers. The figure shows STET allocation technique getting a better performance than by the existing TXOP, RS, HB, TO and AC algorithm allocation technique.

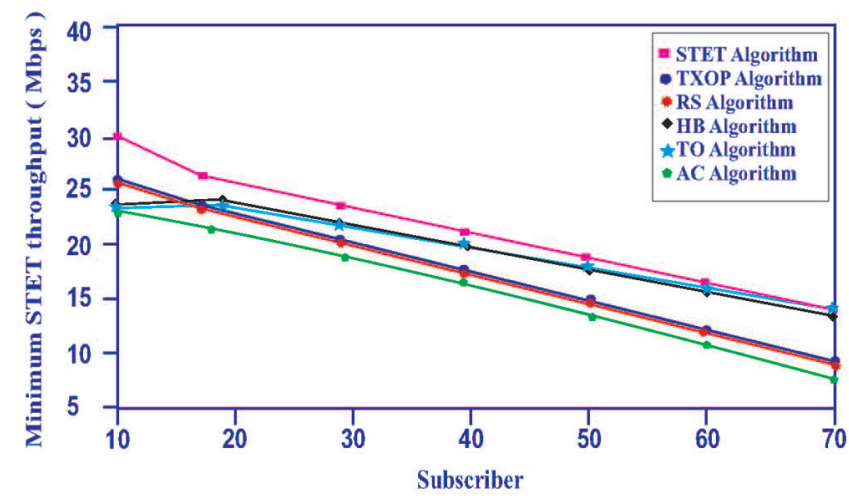

Figure 5: Minimum STET throughput vs. subscriber 


\subsection{Percentage of STET Throughput}

The performance in terms of percentage of STET throughput in the IEEE 802.11ad scenario is shown in Fig. 6.

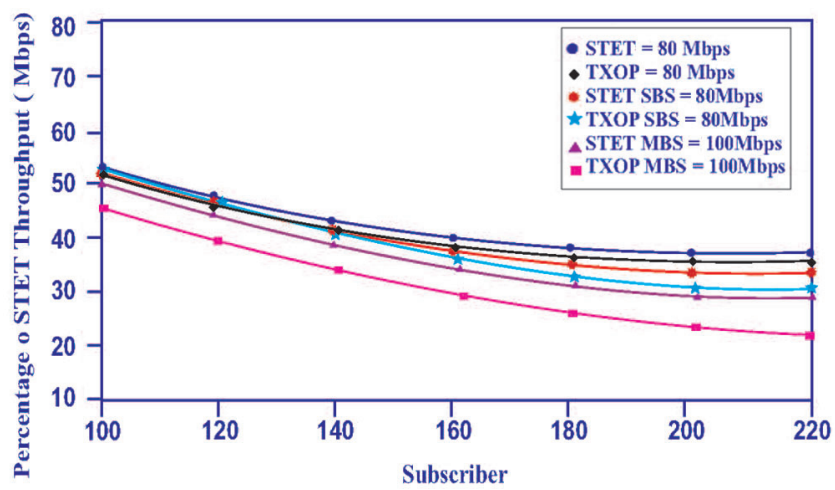

Figure 6: Percentage of STET throughput (Mbps) vs. subscriber

The relationship between the percentage of STET throughput and the number of subscribers, for various 802.11 ad throughput threshold is shown. Support to the maximum numbers is possible when the throughput is minimum. Therefore, STET channel allocation always yields the throughput of a better percentage for the maximum number of subscribers. Fig. 6 shows the possibility of improvement in the percentage of throughput with the use of the STET allocation techniques.

\section{Conclusion}

In this paper, we have presented a mathematical model-based IEEE 802.11ad for the evaluation of the performance in WLAN. STET allocation technique has achieved throughput improvement in voice, video and image transmissions. Moreover, STET allocation technique has helped improvement in usage of the frequency band. The technique has been adopted in the 802.11ad for accessing the MLMU-MIMO communication at medium access control layer level. Riverbed model simulation tool 17.5 has been used for obtaining mathematical results. It gives an idea of the benefit of using the STET allocation technique for maximization of the usage of the rare wireless spectrum and of the medium access fairness between the various access points.

Acknowledgement: Our sincere thanks to our institution management for their constant motivation and encouragement to the publication of our research ideas in this esteemed journal. We thank Professor Dr. T. R. Ganesh babu for leading us by suggesting new ideas in framing our work. Our heartfelt thanks to Dr. J.V Anand for his constant support in bringing out this research work with good findings.

Funding Statement: The author received no specific funding for this study.

Conflicts of Interest: The authors declare that they have no conflicts of interest to report regarding the present study.

\section{References}

[1] J. G. Andrews, S. Buzzi, W. Choi, S. V. Hanly, A. Lozano et al., "What will 5G be?," IEEE Journal on Selected Areas in Communications, vol. 32, no. 6, pp. 1065-1082, 2014. 
[2] M. Shafi, A. F. Molisch, P. J. Smith, T. Haustein, P. Zhuet et al., "5G: A tutorial overview of standards, trials, challenges, deployment and practice," IEEE Journal on Selected Areas in Communications, vol. 35, no. 6, pp. 1201-1221, 2017.

[3] T. Rappaport, S. Sun, R. Mayzus , H. Zhao, Y. Azar et al., "Millimeter wave mobile communications for 5G Cellular: It will work," IEEE Access, vol. 1, no. 1, pp. 335-349, 2013.

[4] K. Sakaguchi, T. Haustein, S. Barbarossa, E. C. Strinati, A. Clemente et al., "Where, When, and How mmwave is used in 5G and Beyond," IEICE Transactions on Electronics, vol. E100.C, no. 10, pp. 790-808, 2017.

[5] A. M. Hamed and R. K. Rao, "Spectral and energy efficiencies in mmwave cellular networks for optimal utilization," Wireless Communications and Mobile Computing, vol. 2018, pp. 11, 2018.

[6] IEEE Std 802.11ad-2012, “(Amendment to IEEE Std 802.11-2012, as Amended by IEEE Std 802.11ae-2012 and IEEE Std 802.11aa-2012. Part 11: Wireless LAN Medium Access Control (MAC) and Physical Layer (PHY) specifications Amendment 3: Enhancements for very high throughput in the $60 \mathrm{GHz}$ Band, pp. 1-628, 2012.

[7] T. Nitsche, C. Cordeiro, A. B. Flores, E. W. Knightly, E. Perahia et al., "IEEE 802.11ad: Directional $60 \mathrm{GHz}$ communication for multi-gigabit-per-second Wi-Fi," IEEE Communications Magazine, vol. 52, no. 12, pp. 132-141, 2014.

[8] X. Tie, K. Ramachandran and R. Mahindra, "On $60 \mathrm{GHz}$ wireless link performance in indoor environments," in Lecture Notes in Computer Science (including subseries Lecture Notes in Artificial Intelligence and Lecture Notes in Bioinformatics). vol. 7192, pp. 147-157, 2012.

[9] N. Saito, T. Tsukizawa, N. Shirakata, T. Morita, K. Tanaka et al., "A fully integrated 60-GHz CMOS transceiver chipset based on WiGig/IEEE 802.11 ad with built-in self-calibration for mobile usage," IEEE Journal of SolidState Circuits, vol. 48, no. 12, pp. 3146-3159, 2013.

[10] S. J. Huang and S. G. Chen, "A High-throughput Radix-16 FFT processor with parallel and normal input/output ordering for IEEE 802. 15.3c systems," IEEE Transactions. Circuits and Systems-I Regular-Papers IEEE-Transactions on IEEE T Circuits-I, 1549-8328, vol. 59, no. 8, pp. 1752-1765, 2012.

[11] L. Koschel and A. Kortke, "Frequency synchronization and phase offset tracking in a real-time 60-GHz CSOFDM MIMO system," in Proc. IEEE PIMRC, Sydney, NSW, Australia, pp. 2281-2286, 2012.

[12] S. Li, G. Yue, X. Cheng and Z. Luo, "A novel and robust timing synchronization method for SC-FDE $60 \mathrm{GHz}$ WPAN systems," in Proc. ICCT, China, pp. 262-267, 2012.

[13] F. C. Yeh, T. Y. Liu, T. C. Wei, W. C. Liu and S. J. Jou, "A SC/OFDM dual mode frequency-domain equalizer for $60 \mathrm{GHz}$ Multi-Gb/s wireless transmission," in Proc. VLSI-DAT, Hsinchu City 300, Taiwan, pp. 1-4, 2011.

[14] S. W. Yen, S. Y. Hung, C. L. Chen, H. C. Chang, S. J. Jou et al., "A 5.79-Gb/s energy-efficient multirate LDPC CODEC chip for IEEE 802.15.3c applications," IEEE Journal of Solid-State Circuits, vol. 47, no. 9, pp. 2246-2257, 2012.

[15] K. Huang and Z. Wang, Millimeter Wave Communication Systems. Wiley, 2011. [Online]. Available: https://www. wiley.com/en-us/Millimeter+Wave+Communication+Systems-p-9780470404621.

[16] Y. I. Nechayev, X. Wu and Constantinou, "Millimetre-wave path-loss variability between two body-mounted monopole antennas," IET Microwaves, Antennas \& Propagation, vol. 7, no. 1, pp. 1-7, 2013.

[17] S. K. Yong, P. Xia and A. Valdes-Garcia, "60 GHz technology for Gbps WLAN and WPAN: From theory to practice. Wiley, 2011. [Online]. Available: https://www.wiley.com/en-in/60GHz+Technology + for + Gbps +WLAN+and+WPAN\%3A+From+Theory+to+Practice-p-9781119956747.

[18] S. Singh, F. Ziliotto and U. Madhow, "Blockage and directivity in $60 \mathrm{GHz}$ wireless personal area networks: From cross-layer model to multihop MAC design," IEEE Journal on Selected Areas in Communications, vol. 27, no. 8, pp. 1400-1413, 2009.

[19] E. Charfi, L. Chaari and L. Kamoun, "PHY/MAC enhancements and QoS mechanisms for very high throughput WLANs: A survey," IEEE Communications Surveys and Tutorials, vol. 15, no. 4, pp. 1714-1735, 2013.

[20] W. Roh, J. Y. Seol, J. Park, B. Lee, J. Lee et al., "Millimeter-wave beamforming as an enabling technology for 5G cellular communications: Theoretical feasibility and prototype results," IEEE Communications Magazine, vol. 52, no. 2, pp. 106-113, 2014. 
[21] C. Dehos, J. L. Gonzalez, A. D. Domenico, D. Kténas, L. Dussop et al., "Millimeter-wave access and backhauling: The solution to the exponential data traffic increase in $5 \mathrm{G}$ mobile communications systems," IEEE Communications Magazine, vol. 52, no. 9, pp. 88-95, 2014.

[22] IEEE 802.11ac/D7.0, "Wireless LAN Medium Access Control (MAC) and Physical Layer (PHY) specifications: Enhancements for very high throughput for operation in bands below $6 \mathrm{GHz}, 2013$.

[23] O. Bejarano, E. W. Knightly and M. Park, "IEEE 802.11ac: From channelization to multi-user MIMO," IEEE Communications Magazine, vol. 51, no. 10, pp. 84-90, 2013.

[24] B. Bellalta, J. Barcelo, D. Staehle, A. Vinel and M. Oliver, "On the performance of packet aggregation in IEEE 802.11ac MU-MIMO WLANs," IEEE Communications Letters, vol. 16, no. 10, pp. 1588-1591, 2012.

[25] C. Zhu, Y. Kim, O. Aboul Magd and C. Ngo, "Multi-user support in next generation wireless LAN," in Proc. CCNC IEEE, Las Vegas, NV, USA, pp. 1120-1121, 2011.

[26] IEEE 802.11ac/D1.0, "Wireless LAN Medium Access Control (MAC) and physical Layer (PHY) specifications: Enhancements for very high throughput for operation in bands below $6 \mathrm{GHz}, " 2012$.

[27] C. Zhu, A. Bhatt, Y. Kim, O. Aboul-Magd and C. Ngo, "MAC enhancements for down-link multi-user MIMO transmission in next generation WLAN," in Proc. CCNC IEEE, Las Vegas, NV, USA, pp. 832-837, 2012.

[28] C. Zhu, C. Ngo, A. Bhatt and Y. Kim, "Enhancing WLAN back-off procedures for downlink MU-MIMO support," in Proc. WCNC IEEE: MAC, Shanghai, China, pp. 368-373, 2013.

[29] G. Bianchi, "Performance analysis of the IEEE 802.11 Distributed Coordination Function," IEEE Journal on Selected Areas in Communications, vol. 18, no. 3, pp. 535-547, 2000. 